\title{
Which are the limiting factors in lung tissue sampling and diagnostic accuracy for a new Interventional Pulmonology Unit? From expert consensus-based evidence to results of a new-born Unit
}

\author{
Cristiano Carbonelli ${ }^{\wedge} \wedge$, Antonio Rossi ${ }^{2}$, Gianluca Ciliberti ${ }^{1}$, Maria Arcangela Grimaldi ${ }^{1}$, \\ Stefano Notarangelo ${ }^{1}$, Paola Parente ${ }^{3}$, Massimiliano Copetti ${ }^{4}$, Alessandro Zanforlin ${ }^{5}$, Filippo Lococo ${ }^{6}$, \\ Marco Taurchini ${ }^{7}$, Evaristo Maiello ${ }^{2}$, Salvatore De Cosmo ${ }^{1}$, Paolo Graziano ${ }^{3}$ \\ ${ }^{1}$ Department of Medical Sciences, Fondazione IRCCS “Casa Sollievo della Sofferenza”, San Giovanni Rotondo, (FG), Italy; ${ }^{2}$ Oncology Unit, \\ Fondazione IRCCS “Casa Sollievo della Sofferenza”, San Giovanni Rotondo, (FG), Italy; ${ }^{3}$ Pathology Unit, Fondazione IRCCS “Casa Sollievo \\ della Sofferenza”, San Giovanni Rotondo, (FG), Italy; ${ }^{4}$ Unit of Biostatistics, Fondazione IRCCS “Casa Sollievo della Sofferenza”, San Giovanni \\ Rotondo, (FG), Italy; ${ }^{5}$ Medicina Interna Ospedale Centrale di Bolzano, Bolzano, Italy; ${ }^{6}$ Department of Thoracic Surgery, Fondazione Policlinico \\ Universitario “A. Gemelli”, IRCCS, Università Cattolica del Sacro Cuore, Rome, Italy; ${ }^{7}$ Thoracic Surgery Unit, Fondazione IRCCS “Casa Sollievo \\ della Sofferenza", San Giovanni Rotondo, (FG), Italy \\ Contributions: (I) Conception and design: C Carbonelli, A Rossi; (II) Administrative support: C Carbonelli; (III) Provision of study materials or \\ patients: S Notarangelo, MA Grimaldi, G Ciliberti; (IV) Collection and assembly of data: P Parente, P Graziano; (V) Data analysis and interpretation: \\ M Copetti, A Zanforlin; (VI) Manuscript writing: All authors; (VII) Final approval of manuscript: All authors. \\ Correspondence to: Cristiano Carbonelli. Department of Medical Sciences, Fondazione IRCCS “Casa Sollievo della Sofferenza”, Viale Cappuccini snc, \\ San Giovanni Rotondo 71013, Italy. Email: c.carbonelli@operapadrepio.it.
}

Background: There is a gap of knowledge about the factors that may determine the quality and the accuracy of diagnostic bronchoscopic procedures when setting up a new Interventional Pulmonology Unit. As little evidence-based medicine is available on this matter, an online consensus opinion of experts was gathered and compared with real-life data coming from a new Interventional Pulmonology (IP) Unit.

Methods: A survey was emailed to the heads of all Italian IP Units to investigate the factors influencing the success of the diagnostic yield of a new IP Unit. The survey consisted of 24 items grouped by topic; the level of agreement ranged from 1 (no influence) to 7 (strong influence). After responses were collected, we submitted the data on the accuracy of the endoscopic procedures performed during the first two years of our new IP Unit to the attention of the participants for a second round of survey; the level of consistency between the first and second round of responses was assessed.

Results: After having been shown the results of the first two years of activity of our Unit, in the second round of the survey the responders indicated the personal skills of the Interventional Pulmonologist, the availability of echoendoscopic technology and the expertise in evaluating cytological samples as the factors able to positively influence the performance of a newly established IP Unit. Neither the role of dedicated nursing assistance, the availability of a rapid on-site evaluation, nor the presence of anesthesiology assistance were considered to be limiting factors for the final accuracy results.

Conclusions: A consensus of opinion of a group of expert interventional pulmonologists highlighted the factors that may be responsible for the diagnostic success of a newly established Italian IP Unit. These factors are mainly three: personal skills of the interventional pulmonologist, the availability of echoendoscopic technology, and the expertise in reading cytological samples.

Keywords: Decision analysis; diagnostic bronchoscopy; echo-endoscopy; organization implementation; quality management; survey

\footnotetext{
^ ORCID: 0000-0002-3083-8387.
} 
Submitted Oct 12, 2020. Accepted for publication Feb 06, 2021.

doi: 10.21037/jtd-20-2990

View this article at: http://dx.doi.org/10.21037/jtd-20-2990

\section{Introduction}

Transbronchial biopsy (TBB) and transbronchial needle aspiration (TBNA) are well-established but still underutilized techniques (1). With the advent of the endobronchial ultrasound-guided transbronchial needle aspiration (EBUS-TBNA), ultrasound became widely used by the interventional community (2). Today, the bronchoscopic mediastinal sampling should be the initial approach to achieve a diagnosis and to stage lung cancer patients as it requires fewer invasive tests and has fewer complications compared to traditional transthoracic or surgical sampling techniques (3). A survival benefit in patients who received EBUS-TBNA in their diagnostic and staging management has also been demonstrated (4). However, an epidemiological study showed that the diagnostic evaluation was consistent with the applicable guidelines for only $21 \%$ of patients, and mediastinal sampling was performed before treatment only on $56 \%$ of patients with NSCLC (5).

The starting point for a correct diagnosis is the adequacy of the bronchoscopic sample. However, the quality and the results of diagnostic bronchoscopic procedures may be affected by other potentially critical factors, such as interprofessional and multidisciplinary organizational factors. This is risk is particularly important to consider when establishing a new Interventional Pulmonology (IP) Unit in a hospital not providing such techniques.

What are the factors determining the success of an IP Unit? The ability of professionals to perform endoscopy; the experience of pathologists in reading cytological or histological samples; the interprofessional elements, such as the competence and the expertise of the nursing team; or the availability of anesthetic assistance or specific endoscopic instrumentation?

With the aim to contribute to this debate, since no evidence was available on this topic in the literature, we designed a Delphi-based analysis to obtain the best expert opinion available within the Italian IP community.

The first phase of the analysis consisted in sending out to all the heads of the Italian IP Units an invitation to take part in a survey designed to inquire their opinion on a set of factors commonly considered crucial in determining the possibility of success for a new IP Unit. After obtaining the first round of answers, we showed the participants the real-life data obtained from the study of the accuracy of the endoscopic procedures performed during the first two years of activity of our new IP Unit.

Then we invited the participants to revise their previous answers in light of the real-life data. On the basis of the degree of agreement in this second round, we draw some conclusions on the factors that may be responsible for the success of diagnostic procedures in a new IP Unit. We present the following article in accordance with the SURGE reporting checklist (available at http://dx.doi.org/10.21037/ jtd-20-2990).

\section{Methods}

We invited the heads of all Italian IP Units to complete a survey distributed via email. The survey consisted of 24 questions related to the possible factors that are considered to be more frequently linked to the outcome results of new IP Units. The responders could indicate the level of importance of each factor by using a modified likelihood scale ranging from 1 (no influence) to 7 (strong influence). The survey was subdivided into 6 groups of questions, each related to a specific topic: (I) factors related to interventional pulmonologists (7 items); (II) factors related to the nursing component (3 items); (III) factors related to pathological assistance (7 items); (IV) factors related to anesthesiology assistance (2 items); (V) factors related to technology availability (4 items); (VI) factors related to the presentation of the disease (1 item). The anonymous experts' responses were shared with the participants and discussed with them. Then we showed the respondents the organizational methods and the performance outcome data of our Unit during the first two years of activity. On the basis of this real-life data, the respondents were given the opportunity to re-evaluate and change their responses to the survey. The level of consistency between the first and second round of responses was assessed. The study protocol, relating to the retrospective measurement of the performance of diagnostic procedures and the identification of predictive biomarkers of response to chemotherapy, was approved by Ethics Committee of the Casa Sollievo della Sofferenza Research Hospital in San Giovanni Rotondo, Italy (No.: ICF/IP 
V1.0_03 Oct 19). Written informed consent was obtained from all participants.

\section{Statistical analysis}

Categorical variables were reported as frequency and percentage. Adequacy and diagnosis were reported as percentage, overall and by subgroups. Adequacy and diagnosis were compared among groups using the Fisher exact test. A P value $<0.05$ was considered statistically significant. The $\mathrm{K}$ statistic was computed to measure the agreement between the raters. All statistical analyses were performed using SAS (version 9.4).

\section{Results}

From March to May 2018, 158 invitation emails were sent out to all of the heads of the Italian IP Units. A total of 56 surveys were collected. The results of the two rounds of responses are shown in Table 1.

Factors related to interventional pulmonologists showed a median value of 5.30 ("high impact"). In particular, a high level of importance was given to the expertise of the first operator with a median value of 6.46 and also to the presence of a multidisciplinary discussion of cases (median score of 6.16), while the scientific production of professionals was considered the least significant factor (median value of 4.48).

Factors related to the nursing component showed the best median value of 6.1 between the groups included in the survey. In this group, the availability of dedicated nursing assistance was the most important factor (value 6.43 ) in the respondents' opinion. Factors related to the anatomopathological assistance scored 5.83. In this group, the following items obtained the higher scores: "Availability of a Rapid On Site Evaluation (ROSE)" scored a value of 5.80; the type of materials provided to the pathologists scored a value of 6.21, and personal experience in reading cytological samples scored a value of 6.20; while the lowest score in this group was given to the personal experience in reading surgical samples (value 5.04).

The median value of the survey for factors related to the anesthesiology assistance was 5.55, without significant differences between dedicated anesthesiology assistance (value 5.57) or anesthesia administered directly by the pulmonologists (value 5.54).

Factors related to technological availability showed a median value of 6.02. In detail, the availability of linear and radial EBUS was the most important factor of the explored ones (value 6.64).

Factors related to the presentation of the disease (size and location of lesion sites) for diagnosis and staging showed a median value of 6.07 .

Overall, $17.8 \%$ of the invited colleagues participated in the second round of our Delphi analysis.

After having been shown the results of the first two years of activity of our Unit, the respondents were given the possibility to revise their previous responses. The result of this second round was that only some factors were indicated as capable of positively influence the performance of a newly established IP Unit.

The importance of the operator's expertise passed from a score of 6.36 to a score of 6.90; that of the "Personal experience in reading cytological samples" passed from 6.20 to 7, and that of the "Availability of linear and radial EBUS" passed from 6.64 to 7 . All the other factors indicated in the survey received lower scores. In particular, the "Availability of a discussion in the Lung Unit" of the cases dropped from 6.16 to 3.20 ; the "Availability of dedicated nursing assistance", from 6.43 to 3.20 ; the "Availability of a ROSE", from 5.80 to 2.80; the "Personal experience in reading surgical samples", from 5.04 to 2.80. Even the value of factors related to anesthesiology assistance fell to 3.80 , in the grey area, which suggests that these factors do not represent a non-negotiable condition for obtaining the best chances of success in the establishment of a new IP Unit.

The level of agreement between the first and the second round of survey responses was calculated. In the first round, the $\mathrm{K}$ of agreement was 0.03 (95\% CI, 0.01-0.04); in the second round, it was 0.57 (95\% CI, 0.50-0.65). These two Ks are statistically different with a $\mathrm{P}$ value $<0.001$.

Demographic and clinical patients' characteristics in the first two years of activity in our new Unit are reported in Table 2. Table 3 shows the diagnostic performance of interventional procedures in our Unit, which started its activity on January 2017 and collected 331 consecutive patients who received interventional bronchoscopic procedures: 217 EBUS TBNA, 25 conventional TBNA and 89 TBB.

\section{Discussion}

The purpose of the study was to identify the non-negotiable factors responsible for diagnostic and tissue sampling accuracy in a newly established IP Unit.

As little evidence was available in the literature on this 


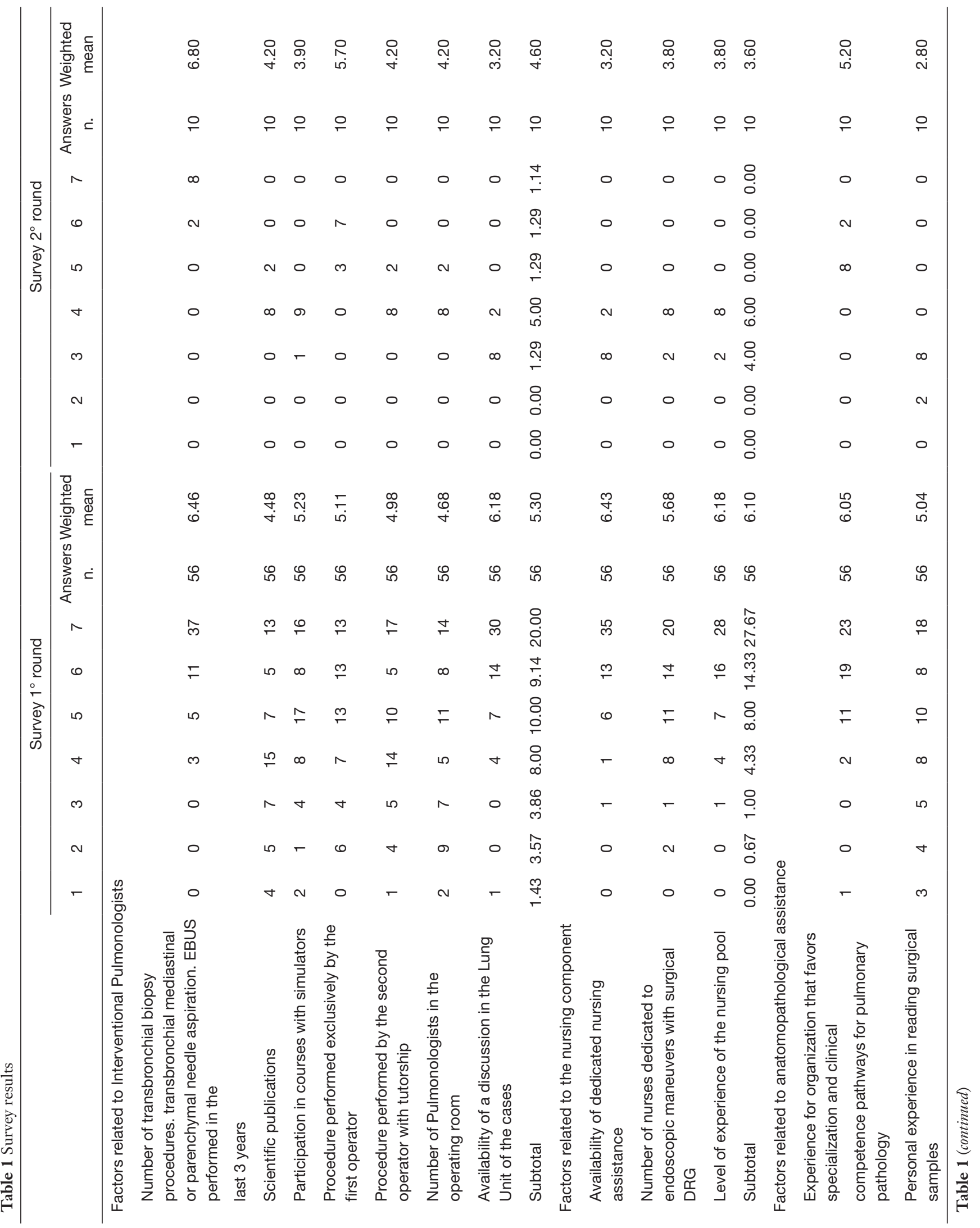




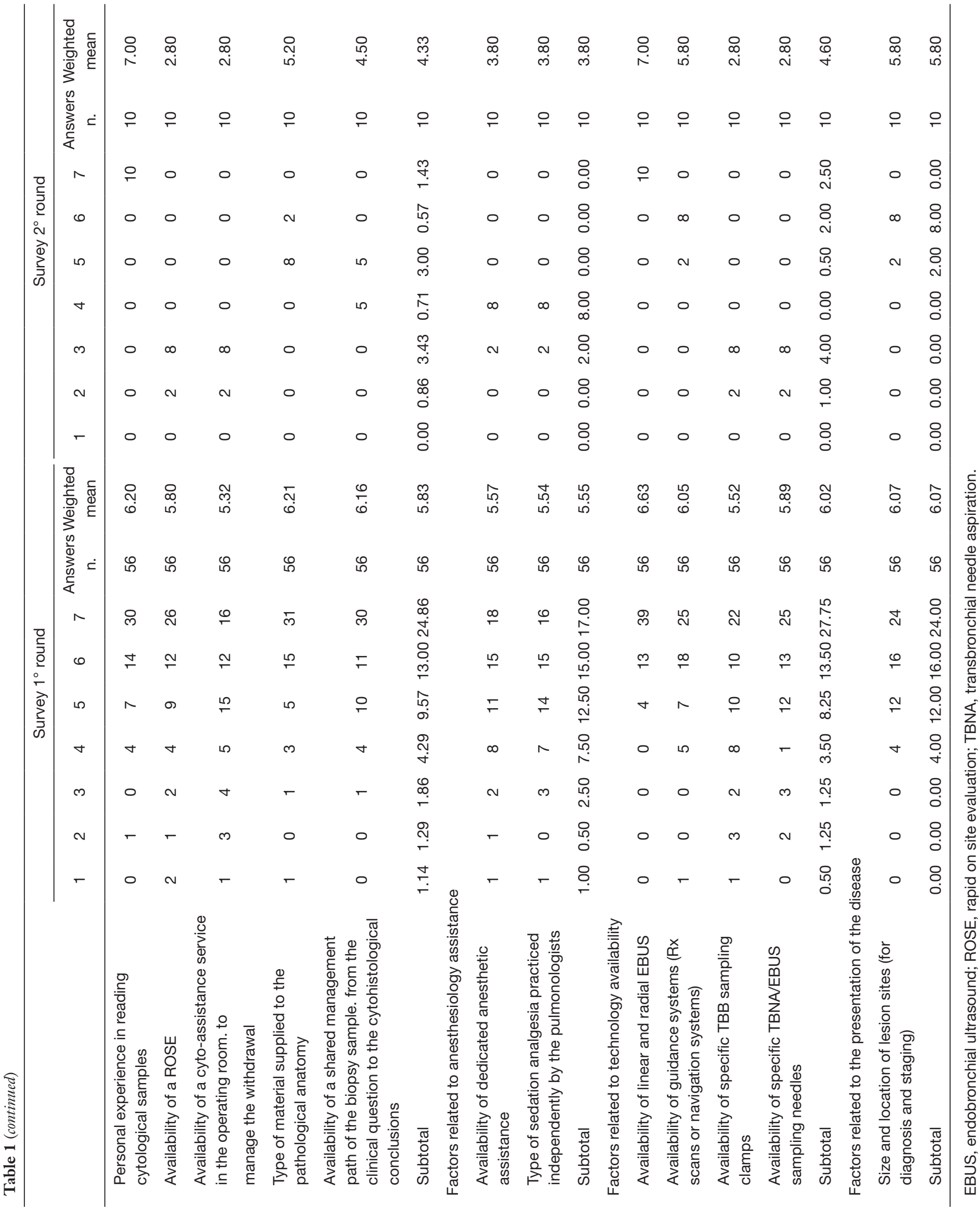


Table 2 Demographical and clinical patients' characteristics

\begin{tabular}{|c|c|}
\hline Variables & Data \\
\hline Number of patients & 331 \\
\hline Median age (range), years & $66.71 \pm 13.68$ \\
\hline \multicolumn{2}{|l|}{ Sex } \\
\hline Male (\%) & $226(68.3)$ \\
\hline Female (\%) & $105(31.7)$ \\
\hline Smoking habit yes (\%) & $48 \%$ \\
\hline \multicolumn{2}{|l|}{ Hospital Unit requesting (\%) } \\
\hline Department of Medical Sciences & $129(39.2)$ \\
\hline Unit of Thoracic Surgery & $110(33.2)$ \\
\hline Unit of Oncology & $58(17.2)$ \\
\hline Unit of Geriatrics & $11(3.3)$ \\
\hline Other Hospitals & $6(1.8)$ \\
\hline Unit of Gastroenterology & $5(1.5)$ \\
\hline Unit of Neurosurgery & $3(0.9)$ \\
\hline Unit of Hematology & $3(0.9)$ \\
\hline Unit of Orthopedic & $3(0.9)$ \\
\hline Others & $3(0.9)$ \\
\hline \multicolumn{2}{|l|}{ Clinical motivation (\%) } \\
\hline Diagnosis & $279(84.3)$ \\
\hline Staging & $33(10.0)$ \\
\hline Re-biopsy & $10(3.0)$ \\
\hline Re-staging & $9(2.7)$ \\
\hline \multicolumn{2}{|l|}{ Iconography (\%) } \\
\hline Lung opacification & $47(14.2)$ \\
\hline Lung opacification + adenopathies & $29(8.8)$ \\
\hline Adenopathies & $120(36.3)$ \\
\hline Interstitial lung diseases & $16(4.8)$ \\
\hline Multiple nodules & $3(0.9)$ \\
\hline Nodule $<2 \mathrm{~cm}$ & $14(4.4)$ \\
\hline Nodule $<2 \mathrm{~cm}+$ adenopathies & $24(7.3)$ \\
\hline Nodule $>2 \mathrm{~cm}$ & $18(5.4)$ \\
\hline Nodule $>2 \mathrm{~cm}+$ adenopathies & $60(18.1)$ \\
\hline
\end{tabular}

Table 2 (continued)
Table 2 (continued)

\begin{tabular}{|c|c|}
\hline Variables & Data \\
\hline \multicolumn{2}{|l|}{ Procedures (\%) } \\
\hline EBUS & $217(65.6)$ \\
\hline TBB & $51(15.4)$ \\
\hline $\begin{array}{l}\text { TBB + assisted by guidance systems (virtual } \\
\text { bronchoscopy, radioscopy or echoendoscopy) }\end{array}$ & $38(11.5)$ \\
\hline TBNA & $25(7.6)$ \\
\hline \multicolumn{2}{|l|}{ Number of physicians } \\
\hline 1 & $125(38.0)$ \\
\hline 2 & $206(62.0)$ \\
\hline \multicolumn{2}{|l|}{ First operator } \\
\hline Expert & $231(69.8)$ \\
\hline Less expert & $100(30.2)$ \\
\hline \multicolumn{2}{|l|}{ Anesthesiology assistance (\%) } \\
\hline No & $316(95.5)$ \\
\hline Yes & $15(4.5)$ \\
\hline \multicolumn{2}{|l|}{ Diagnosis } \\
\hline ADK & $111(33.4)$ \\
\hline Squamous carcinoma & $25(7.5)$ \\
\hline CANOS & $1(0.3)$ \\
\hline Poorly differentiated NSCLC & $2(0.6)$ \\
\hline Metastasis & $23(6.9)$ \\
\hline Small cell lung cancer & $22(6.6)$ \\
\hline Organizing pneumonia & $10(3.0)$ \\
\hline Sarcoid & $20(6.0)$ \\
\hline Lymphatic tissue & $63(19.0)$ \\
\hline IgG4 related disease & $1(0.3)$ \\
\hline Tuberculosis & $5(1.5)$ \\
\hline Carcinoid & $1(0.3)$ \\
\hline Smoking related lung diseases & $5(1.5)$ \\
\hline Pneumocyte hyperplasia & $1(0.3)$ \\
\hline IPF & $1(0.3)$ \\
\hline Mesothelioma & $1(0.3)$ \\
\hline Lymphoma & $5(1.5)$ \\
\hline LIP & $1(0.3)$ \\
\hline Lung inflammation & $16(4.8)$ \\
\hline Alveolar haemorrhage & $4(1.2)$ \\
\hline Inadequate & $13(3.9)$ \\
\hline
\end{tabular}


Table 3 Adequacy and diagnosis according to procedures, overall and by subgroups

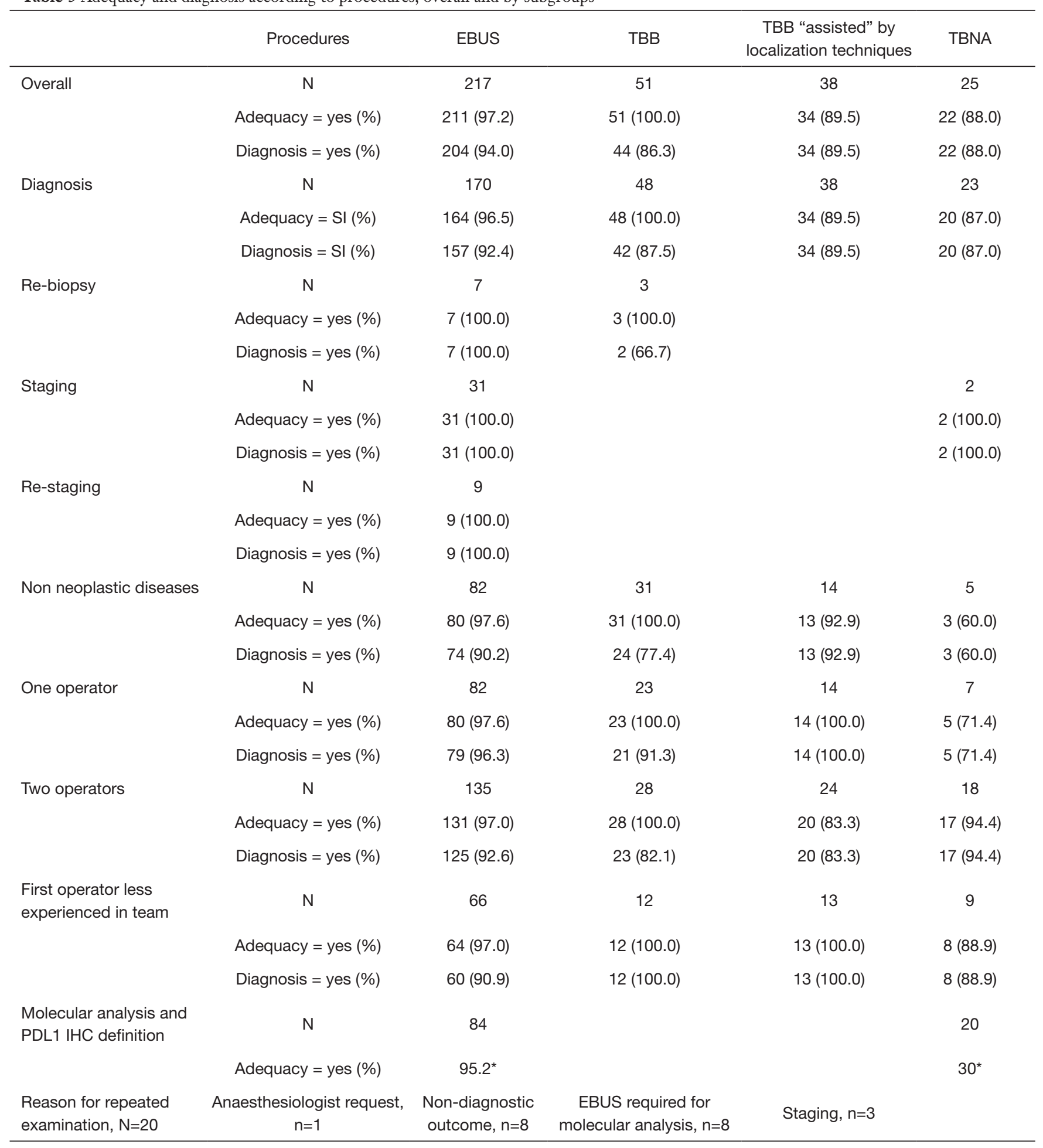

${ }^{*} \mathrm{P}<0.0001$. EBUS, endobronchial ultrasound; TBB, transbronchial biopsy; TBNA, transbronchial needle aspiration. 
matter, we used the Delphi method to obtain the most reliable consensus of opinion by surveying a group of Italian IP experts. The participants were sent out a questionnaire in two rounds interspersed with controlled opinion feedback, which in this case was represented by the presentation of real-life data. A limitation of our study is the low rate of responses to the second round of the survey, probably due to the fact that in Italy there is little interest in participating in this kind of surveys.

The results of the study show that there is a wide consensus among the experts that technology (especially linear and radial EBUS) may influence the accuracy of endoscopic procedures.

There is a certain body of evidence suggesting that the availability of advanced EBUS technology improves diagnostic adequacy and the achievement of molecular profiling (6). In our experience, the availability of radioscopy and EBUS, both radial and linear, improved the diagnostic yield, especially regarding the results of the molecular characterization of pulmonary neoplasms.

Factors relating to dedicated nursing assistance were given high importance during the first round of survey, even though there is no scientific literature supporting this result. In our practice, for example, our operating room nursing staff had no previous experience in thoracic endoscopy and, when in 2017, the endoscopic activity was also assigned to the operating room of our Unit, the nursing staff continued to rotate daily to perform other low intensity activities relating to thoracic, abdominal and dermatologic surgery, without any loss in quality when performing the endoscopyrelated activities.

The training provided to the nurses was both on the phases of endoscopic assistance and the reprocessing of the instruments. It was conducted by medical operators, biomedical technicians of the hospital and endoscopic instruments manufacturers.

About the anatomopathological assistance, the results of the survey revealed that the respondents placed great importance on the following factors: the type of biological material supplied to the pathologists and the operator's expertise in reading cytological samples. At present, there is no consensus among pathologists on whether cell block should be preferred over smears $(7,8)$. The current guidelines do not recognize a diagnostic superiority of the cell block over smears, nor they recommend rapid on site evaluation (ROSE) for every procedure; on the contrary, they suggest leaving this decision to the operator's discretion $(9,10)$.
In our hospital, neither cytology assistance nor ROSE were available, and smears were not provided to the pathologists. As a consequence, in order to achieve the best diagnostic accuracy possible, a shared management of the histologic and cytological sampling was agreed, and cell blocks were always provided when a TBNA or an EBUS procedure were performed.

The second round of survey revealed that, according to the respondents, the experience gained by the operator in reading cytological samples is a key factor. The literature confirms that some improvements are achieved in the pathologists' learning curve once they become more familiar with reading cytological EBUS or EUS (endoscopic ultrasound) specimens $(11,12)$. In our hospital, the negative effects of a physiological inter-professional learning curve for reading the anatomopathological samples were minimized thanks to a continuous quality control of the anatomopathological reading of the sample along with a shared management path of the biopsy sample, from the clinical question to the cyto-histological conclusions.

The request for cytohistological analysis was therefore sent to the pathology service and included the clinical information requested by the pathologists in order to be able to automatically proceed to the first immunohistochemical analysis to establish the target therapies in cases of squamous cell disease and adenocarcinoma. If necessary, further molecular analysis was started. The information requested by the pathology service were the following: a patient's tobacco history; clinical suspicion of primitiveness; and the disease stage.

About the factor related to interventional pulmonologists, the literature suggests that there is a lack of specific training in using mediastinal transbronchial sampling techniques (5). High volume hospitals have better diagnostic yields and lower complication rates for EBUS-TBNA (13) than hospitals doing less regular examinations.

The learning curves of physicians for the execution of the abovementioned procedures improves as specific training through the traditional patient-based apprenticeship model and simulation based educational programs is done $(14,15)$.

In our Unit, the procedures were performed by one senior interventional pulmonologist, one trainee doctor, and two nurses. The presence of the second medical operator, who had previously attended specific postgraduate or simulation-based courses, was requested by the training program. In our Lung Unit, multidisciplinary team meetings to discuss patient cases were regularly organized to decide upon the appropriate treatment but 
rarely upon the appropriate diagnostic procedure to apply. Our experience confirms the opinion of the majority of the colleagues involved in the survey. Our acceptable diagnostic results were due almost exclusively to the presence of the expert operator (95\%) in the operating room but not to his/ her performing of the test, given that the trainee doctor was the first operator in $30 \%$ of cases.

There is no evidence to recommend any type of anesthesia (16), and the available guidelines do not report better performances in the presence of anesthesiological assistance.

When the results of clinical trials describing the clinical outcomes of systematic mediastinal staging are available (17), it will be necessary to assess whether to make greater use of the anesthesiological assistance.

In our experience, complex endoscopic activity was performed in a general operating room. Patients received a conscious sedation protocol with intravenous midazolam and fentanyl. Only occasionally the procedures were performed with anesthesia care and laryngeal mask.

\section{Conclusions}

A consensus of opinion of a group of expert interventional pulmonologists highlighted the factors that may be responsible for the diagnostic success of a newly established Italian IP Unit. These factors are mainly three: personal skills of the interventional pulmonologist, the availability of echoendoscopic technology, and the expertise in reading cytological samples. All of the other factors included in the survey, such as dedicated nursing assistance, the availability of a rapid on-site evaluation, and the presence of anesthesiology assistance, resulted to be almost uninfluential.

Although the results of this opinion-based study should be validated by evidence-based medicine to gain scientific validity, its ultimate objective was to pave the way for further research in a field which has never been explored so far and that may shed light on possible useful implications, such as a better allocation of economical and human resources when the factors responsible for the operational success of a new IP Units are well defined.

\section{Acknowledgments}

To Mrs. Chiara Di Giorgio for linguistic editing and to the nursing team: Mrs. Emanuela Coco, Mr. Michele Carriera, Mrs. Costantina Di Lella, Mrs. Anna Vittoria Impagliatelli,
Mrs. Anna Maria Maiolino, Mrs. Concetta Merla, Mrs. Oriana Minchillo, Mrs. Maria Olivieri, Mr. Matteo Tavaglione, Mr. Nicola Tancredi, Mrs. Rosa Merla, Mrs. Arcangela Cappelli, Mrs. Incoronata Occhiochiuso, Mrs. Luigia Russo, Mrs. Lucia Baorda, Mrs. Lucia Dragano, and Mrs. Consiglia Merlino.

Funding: None.

\section{Footnote}

Reporting Checklist: The authors have completed the SURGE reporting checklist. Available at http://dx.doi. org/10.21037/jtd-20-2990

Data Sharing Statement: Available at http://dx.doi. org/10.21037/jtd-20-2990

Peer Review File: Available at http://dx.doi.org/10.21037/jtd20-2990

Conflicts of Interest: All authors have completed the ICMJE uniform disclosure form (available at http://dx.doi. org/10.21037/jtd-20-2990). The authors have no conflicts of interest to declare.

Ethical Statement: The authors are accountable for all aspects of the work in ensuring that questions related to the accuracy or integrity of any part of the work are appropriately investigated and resolved. The study was conducted in accordance with the Declaration of Helsinki (as revised in 2013). The study protocol, relating to the retrospective measurement of the performance of diagnostic procedures and the identification of predictive biomarkers of response to chemotherapy, was approved by Ethics Committee of the Casa Sollievo della Sofferenza Research Hospital in San Giovanni Rotondo, Italy (No.: ICF/IP V1.0_03 Oct 19). Written informed consent was obtained from all participants.

Open Access Statement: This is an Open Access article distributed in accordance with the Creative Commons Attribution-NonCommercial-NoDerivs 4.0 International License (CC BY-NC-ND 4.0), which permits the noncommercial replication and distribution of the article with the strict proviso that no changes or edits are made and the original work is properly cited (including links to both the formal publication through the relevant DOI and the license). See: https://creativecommons.org/licenses/by-nc-nd/4.0/. 


\section{References}

1. Ost DE, Ernst A, Lei X, et al. Diagnostic Yield and Complications of Bronchoscopy for Peripheral Lung Lesions Results of the AQuIRE Registry Am J Respir Crit Care Med 2016;193:68-77.

2. Wahidi MM, Yasufuku K. Point: Should Endobronchial Ultrasound Guide Every Transbronchial Needle Aspiration of Lymph Nodes? Yes. Chest 2013;144:732-4.

3. Ost DE, Yeung SCJ, Tanoue LT, et al. Cinical and organizational factors in the initial evaluation of patients with lung cancer: diagnosis and management of lung cancer, 3rd ed: American College of Chest Physicians evidence-based clinical practice guidelines. Chest 2013;143:e121S-e141S.

4. Navani N, Nankivell M, Lawrence DR, et al. Lung cancer diagnosis and staging with endobronchial ultrasoundguided transbronchial needle aspiration compared with conventional approach: an open-label, pragmatic, randomised controlled trial. Lancet Respir Med 2015;3:282-9.

5. Ost DE, Niu J, Elting LS, et al. Determinants of practice patterns and quality gaps in lung cancer staging and diagnosis. Chest 2014;145:1097-113.

6. Czarnecka-Kujawa K, Yasufuku K. Molecular alterations in non-small-cell lung cancer: perspective for targeted therapy and specimen management for the bronchoscopist. Respirology 2014;19;1117-25.

7. Crapanzano JP, Heymann JJ, Monaco S, et al. The state of cell block variation and satisfaction in the era of molecular diagnostics and personalized medicine. Cytojournal 2014;11:7.

8. Stoy SP, Segal JP, Mueller J, et al. Feasibility of Endobronchial Ultrasound-guided Transbronchial Needle Aspiration Cytology Specimens for Next Generation Sequencing in Non-small-cell Lung Cancer. Clin Lung Cancer 2018;19:230-8.e2.

9. van der Heijden EH, Casal RF, Trisolini R, et al. Guideline for the acquisition and preparation of conventional and endobronchial ultrasound-guided transbronchial needle aspiration specimens for the diagnosis and molecular testing of patients with known or suspected lung cancer. Respiration 2014;88:500-17

10. Wahidi MM, Herth F, Yasufuku K, et al. Technical Aspects of Endobronchial UltrasoundGuided Transbronchial Needle Aspiration: CHEST Guideline and Expert Panel
Report. Chest 2016;149:816-35.

11. Tremblay A, Stather DR, MacEachern P, et al. A randomized controlled trial of standard vs endobronchial ultrasonography-guided transbronchial needle aspiration in patients with suspected sarcoidosis. Chest 2009; 136:340-6.

12. Skov BG, Baandrup U, Jakobsen GK, et al. Cytopathologic Diagnoses of Fine-Needle Aspirations From Endoscopic Ultrasound of the Mediastinum Reproducibility of the Diagnoses and Representativeness of Aspirates From Lymph Nodes. Cancer 2007;111:234-41.

13. Ost DE, Ernst A, Lei X, et al. Diagnostic yield of endobronchial ultrasound-guided transbronchial needle aspiration: results of the AQuIRE Bronchoscopy Registry. Chest 2011;140:1557-66.

14. Fernández-Villar A, Leiro-Fernández V, Botana-Rial M, et al. The endobronchial ultrasoundguided transbronchial needle biopsy learning curve for mediastinal and hilar lymph node diagnosis. Chest 2012;141:278-9.

15. Sehgal IS, Dhooria S, Aggarwal AN. Training and proficiency in endobronchial ultrasoundguided transbronchial needle aspiration: A systematic review. Respirology 2017;22:1547-57.

16. Casal RF, Lazarus DR, Kuhl K, et al. Randomized trial of endobronchial ultrasound-guided transbronchial needle aspiration under general anesthesia versus moderate sedation. Am J Respir Crit Care Med 2015;191:796-803.

17. Bousema JE, Dijkgraaf MGW, Papen-Botterhuis NE, et al. MEDIASTinal staging of non-small cell lung cancer by endobronchial and endoscopic ultrasonography with or without additional surgical mediastinoscopy (MEDIASTrial): study protocol of a multicenter randomised controlled trial. BMC Surg 2018;18:27.

Cite this article as: Carbonelli C, Rossi A, Ciliberti G, Grimaldi MA, Notarangelo S, Parente P, Copetti M, Zanforlin A, Lococo F, Taurchini M, Maiello E, De Cosmo S, Graziano P. Which are the limiting factors in lung tissue sampling and diagnostic accuracy for a new Interventional Pulmonology Unit? From expert consensus-based evidence to results of a new-born Unit. J Thorac Dis 2021;13(5):2942-2951. doi: 10.21037/ jtd-20-2990 